\title{
Tumor-associated edema in brain cancer patients: pathogenesis and management
}

\author{
Roth, P ; Regli, L ; Tonder, M ; Weller, M
}

\begin{abstract}
The long-term treatment of peritumoral edema remains a major challenge in clinical neurooncology. Steroids have been and will remain the backbone of any anti-edematous therapy because of their striking activity, convenient oral administration and also because of their cost-effectiveness. Their side effects, however, can compromise quality of life, particularly upon continuous administration. Therapeutic alternatives which may replace or - at least - help to reduce the steroid dose are limited. However, with the development of new agents such as corticorelin acetate, there is a hope that steroid-induced side effects can be delayed and reduced. The administration of anti-angiogenic agents with steroid-sparing effects, for example, bevacizumab, is limited due to their costs. Increased knowledge on boswellic acids and cyclooxygenase-2 inhibitors which are available for clinical application may help to exploit their anti-edema activity more efficiently in the future.
\end{abstract}

DOI: https://doi.org/10.1586/14737140.2013.852473

Posted at the Zurich Open Repository and Archive, University of Zurich

ZORA URL: https://doi.org/10.5167/uzh-84750

Journal Article

Accepted Version

Originally published at:

Roth, P; Regli, L; Tonder, M; Weller, M (2013). Tumor-associated edema in brain cancer patients: pathogenesis and management. Expert Review of Anticancer Therapy, 13(11):1319-1325.

DOI: https://doi.org/10.1586/14737140.2013.852473 
Tumor-associated edema in brain cancer patients: pathogenesis and management

Patrick Roth $^{1,3^{*}}$, Luca Regli $^{2,3}$, Michaela Tonder ${ }^{1,3}$ and Michael Weller ${ }^{1,3}$

Affiliations:

Departments of ${ }^{1}$ Neurology and ${ }^{2}$ Neurosurgery and ${ }^{3}$ Brain Tumor Center Zurich, University Hospital Zurich, Switzerland

*Correspondence: Dr. Patrick Roth, Department of Neurology, University Hospital Zurich, Frauenklinikstrasse 26, 8091 Zurich, Switzerland, Tel.: +41 (0)44 255 5511, Fax: +41 (0)44 255 4380, E-mail: patrick.roth@usz.ch

Keywords: brain tumor, edema, VEGF, steroids, corticorelin acetate, boswellic acids 


\section{Summary}

The long-term treatment of peritumoral edema remains a major challenge in clinical neurooncology. Steroids have been and will remain the backbone of any anti-edematous therapy because of their striking activity, convenient oral administration and also because of their cost effectiveness. Their side effects, however, can compromise quality of life, particularly upon continuous administration. Therapeutic alternatives which may replace or at least - help to reduce the steroid dose are limited. However, with the development of new agents such as corticorelin acetate, there is hope that steroid-induced side effects can be delayed and reduced. The administration of anti-angiogenic agents with steroid-sparing effects, e.g. bevacizumab, is limited due to their costs. Increased knowledge on boswellic acids and COX-2 inhibitors which are available for clinical application may help to exploit their anti-edema activity more efficiently in the future. 


\section{Background}

Peritumoral edema is a typical finding of malignant neoplasms of the central nervous system (CNS) such as high-grade gliomas and metastases. It can, however, occur in virtually any CNS neoplasm including World Health Organization (WHO) grade I meningiomas, which are among the most common benign brain tumors. Rare subtypes of benign meningiomas such as angiomatous, microcystic and secretory meningioma may be associated with prominent perifocal edema. The presence of edema, therefore, is not a direct indicator of malignancy [1]. Therapeutic interventions on the tumor such as surgery, irradiation or chemotherapy may influence edema evolution [2-4]. Tumor-associated edema significantly contributes to the mass effect of CNS neoplasms and neurological deterioration. The pathophysiological mechanisms leading to the development of edema have been stepwise characterized in the last decades. The improved understanding of the molecular determinants underlying edema formation is a prerequisite for the development of novel therapeutic agents with anti-edema activity.

\section{Pathogenesis}

Peritumoral edema does not necessarily represent a single entity but may differ regarding their pathogenesis between meningiomas, gliomas and metastases. However, several basic mechanisms have been described as hallmarks of peritumoral edema. Similar to edema associated with abscess, tumor-related edema is considered to be vasogenic, that is, disturbed blood-brain barrier function resulting in increased vascular permeability. The altered vascular architecture in brain tumors results in loss of the barrier function and allows for a leakage of plasma fluid and proteins into the surrounding tissue [5]. Such a dysfunctional blood-brain barrier is found in high-grade gliomas but also many other malignant brain tumors. Tumor cells typically produce various cytokines which act on endothelial cells located within or around the tumor. Among others, the most important cytokine secreted by various brain tumors is vascular endothelial growth factor (VEGF) [6]. VEGF has initially been described as vascular permeability factor indicating that it is one of the most potent mediators of vascular permeability [7,8]. Under the influence of VEGF, the permeability of endothelium is increased resulting in a disturbance of the blood-brain barrier $[9,10]$. Tumor-derived VEGF may also enhance blood flow and induce vasodilatation [11]. It binds to different receptors 
which are expressed by endothelial cells and also acts as a critical driver of angiogenesis in various neoplasms [12]. In many tumors such as gliomas, VEGF levels correlate with the grade of malignancy [13]. In meningiomas, the occurrence of brain edema is associated with increased VEGF levels which may contribute to edema evolution [14-16]. Similar mechanisms are present in metastatic brain tumors. They also release angiogenic factors acting on endothelial cells and promoting capillary formations characterized by various abnormalities.

Besides VEGF, other factors which may be involved in the evolution of peritumoral edema comprise arachidonic acid metabolites and nitric oxide (NO). Increased leukotriene $\mathrm{C} 4$ expression was noticed in brain tumors and may contribute to edema development by increasing the blood-brain barrier permeability [17]. Furthermore, the microenvironment surrounding brain tumors may also participate in the evolution of edema. Microglial cells infiltrating brain tumors are characterized by high expression levels of cyclooxygenase-2 (COX-2) which generates prostaglandin E2 (PGE2). COX-2 is also expressed by glioma cells $[18,19]$. COX-2-derived PGE2, in turn, may promote the growth of experimental gliomas and contribute to edema development [20,21]. Similar mechanisms have been claimed for meningioma-associated edema [22]. VEGF-mediated NO expression through induction of endothelial nitric oxide synthase (eNOS) can promote vasodilation in brain tumors [23]. NO production in tumor endothelial cells may subsequently contribute to edema formation but its exact role has not been clarified [24,25].

Tumor vessels are characterized by fenestrations and pathological tight junctions [26,27]. Various molecules which contribute to the function of tight junctions such as claudins and occludin are down-regulated on tumor microvessels [28,29]. Intracellular molecules including Zonula Occludens (ZO)-1 and ZO-2 mediate an interaction of tight junction proteins with the endothelial cell cytoskeleton. Reduced expression levels of these proteins preclude appropriate function of tight junctions and results in fluid extravasation and evolution of edema. Furthermore, the functional activity of occludin and ZO-1 may be impaired by VEGF which subsequently translates into an opening of tight junctions [30].

Is has also been reported that increased glutamate levels secreted via the $\mathrm{xCT}$ transporter which is expressed by glioma cells contribute to the formation of peritumoral edema. Pharmacological or genetic inhibition of the xCT transporter resulted in a reduction of edema and prolonged survival of glioma-bearing rats [31]. Additional molecules which may be involved in the development of peritumoral edema include the family of aquaporins (AQP), a group of water channel proteins. AQPs are integral membrane proteins that are broadly 
expressed in many cells. They are involved in the control of water flow into and out of cells [32]. AQP1 is overexpressed in tumor microvessel endothelia as well as glial and metastatic tumor cells [33]. In a similar study, increased AQP4 levels were observed in high-grade astrocytomas and brain metastases [34]. These authors noticed a correlation between bloodbrain barrier opening and increased AQP4 levels. AQP4 upregulation is also associated with brain edema formation in malignant gliomas [35]. Furthermore, AQP4 protein levels correlate with VEGF expression levels in gliomas [36]. AQP5 is highly expressed in meningiomas and correlates with the intensity of edema [37]. As a consequence, the upregulation of AQP family members may be implicated in the flow of edema fluid. However, their exact role in the evolution and persistence of peritumoral edema needs further investigation.

\section{Management of edema.}

\section{Steroids}

Vasogenic edema typically responds very well to corticosteroid treatment. Therefore, steroids are the most frequently used agents for the treatment of brain tumor-surrounding edema. The first reports, published in the 1950s, suggested a beneficial effect on the outcome of patients undergoing brain tumor surgery when cortisol or other drugs with glucocorticoid activity were administered perioperatively $[38,39]$. The availability of synthetic steroids such as prednisone or dexamethasone resulted in a rapid and widespread use of these agents in brain tumor patients. The mode of action of steroids, their clinical use as well as their important side effects have recently been reviewed in detail $[40,41]$. Therefore, the following section represents only a brief summary on some of the most important aspects of steroids as antiedematous agents. Dexamethasone is used by most neurooncologists because of its high glucocorticoid potency which is required for anti-edema therapy, hardly any mineralocorticoid side effects and a long biological half-life in the range of 48-54 $\mathrm{h}$ allowing for a daily single-dose administration. Hardly any data from placebo-controlled trials assessing the efficacy of steroids on the tumor-surrounding edema in brain tumors are available. However, there is not doubt on their distinct anti-edema activity resulting in a widespread use all over the world. After administration, corticosteroids may result in rapid relief from neurological symptoms due to the reduction of the edematous mass. This effect, however, is typically transient and diminishes within weeks or months. Furthermore, the use 
of steroids may be associated with significant toxicity precluding their unrestricted use. The most frequent acute side effects associated with the administration of steroids include hyperglycemia and arterial hypertension. Long-term intake frequently results in the development of osteoporosis, myopathy, psychiatric alterations, skin thinning and an increased risk for some opportunistic infections. Furthermore, steroids may reduce the sensitivity of tumor cells, other than lymphoma cells, to chemotherapeutic agents. Corticosteroids may also interfere with the metabolism of other therapeutics such as anticonvulsants, and vice versa. Therefore, steroid administration should be minimized whenever possible. However, despite these limitations, glucocorticoids remain the most important component in the treatment of peritumoral edema in brain cancer patients.

\section{Corticorelin acetate}

Human corticotropin-releasing factor (hCRF) is a naturally occurring neuropeptide which is produced in the hypothalamus [42]. It regulates the release of adrenocorticotropin hormone (ACTH) from the pituitary gland which subsequently induces hydrocortisone liberation from the adrenal gland. There are 2 hCRF receptors, CRF1 and CRF2, which are also expressed by numerous tumor cells [43]. Data from experimental glioma models suggest that hCRF may decrease tumor vascular permeability and vasogenic edema in vivo [44,45]. In these experiments, hCRF decreased the peritumoral edema also in adrenalectomized animals, pointing to a mode of action independent of the release of corticosteroids. It has been speculated that the anti-edematous effect of hCRF is due to a direct action on the microvasculature [46]. A phase I trial of hCRF in patients with peritumoral brain edema indicated that the drug is well tolerated when administered as a continuous infusion. Ten out of 17 patients experienced improvement of neurological symptoms whereas rather minor effects on the edema were observed by magnetic resonance imaging (MRI). Clinical improvement did not correlate with changes in cortisol levels [47]. Corticotropin acetate (CrA, Xerecept) is a synthetic analog of hCRF. When administered to glioma-bearing nude mice, it resulted in prolonged survival compared to dexamethasone- or temozolomide-treated animals [48]. The combination of CrA with bevacizumab in a rodent glioma model was associated with prolonged survival compared to either drug alone [49]. A prospective, randomized, double-blind study of 200 patients with primary and secondary brain tumors assessed the putative steroid-sparing effect of CrA. All patients had to be on a stable steroid 
dose at enrolment. Patients within the CrA group received subcutaneous injections of $1 \mathrm{mg}$ of the drug twice daily. CrA was well tolerated with injection site reactions as the only adverse event that was more prevalent in the CrA group. The primary end point, a reduction of the dexamethasone dose by $50 \%$ or more without worsening of neurological symptoms or performance status, showed a trend in favor of the CrA group without reaching statistical significance $(\mathrm{p}=0.12)$. However, the maximum percent reduction of the dexamethasone dose was more pronounced in the CrA group (62.7\%) than in the control group (51.4\%, p<0.001). Furthermore, patients receiving CrA were less frequently affected by myopathy and less likely to experience Cushingoid appearance [50]. Overall, these results are promising and CrA may represent a novel option allowing for a reduction of steroid administration in some patients. However, the steroid-sparing effects of CrA need further investigation. Future trials should define whether CrA administration may allow for a complete replacement of glucocorticoids in patients requiring only small steroid doses.

\section{Anti-angiogenic agents}

As outlined above, the abundant expression of VEGF in various brain tumors which are typically associated with significant perifocal edema has been recognized about 20 years ago [51]. In contrast, tumors which commonly lack large edemas such as pituitary adenomas are characterized by lower VEGF expression. Owing to its implication in the development of peritumoral edema, inhibition of VEGF signaling has been regarded as a promising therapeutic strategy for a long time. However, only within the last years, various agents targeting the VEGF pathway have become widely available.

Bevacizumab is a humanized monoclonal antibody against VEGF-A which has been approved by the FDA for treatment of recurrent glioblastoma. In glioblastoma patients, bevacizumab administration frequently causes a distinct reduction in edema which is commonly associated with a reduction or even complete cessation of steroid administration [52,53]. MR imaging frequently demonstrates a reduction of peritumoral edema upon bevacizumab administration [54]. However, owing to its cost and the lack of approval in this indication, bevacizumab cannot be considered an alternative to steroids. Another agent which has been developed within the last years is the pan-VEGF receptor tyrosine kinase inhibitor cediranib. In a rodent glioma model, cedarinib prolonged survival of tumor-bearing mice due to its anti-edema action without inhibiting the growth of tumor cells proper [55]. Cediranib has also shown 
efficacy in normalization of tumor vessels in recurrent glioblastoma patients and reduction of peritumoral edema [56]. A steroid-sparing effect of cediranib was also reported in a further series of patients with progressive glioblastoma [57]. However, the failure of the compound to prolong progression-free survival in a phase III study in patients with recurrent glioblastoma (NCT00777153), administered either alone or in combination with lomustine, has questioned the clinical activity of the compound [58]. Other novel agents targeting the VEGF pathway such as pazopanib, a multikinase angiogenesis inhibitor may be active against brain tumorsurrounding edema but must currently be considered as an experimental approach in neurooncology [59].

\section{Boswellic acids}

Boswellic acids, phytotherapeutic agents which are obtained by extraction from boswellia serrata, have been assessed for their anti-inflammatory properties and as putative anti-cancer drugs for many years. Boswellic acids are cytotoxic to glioma cells in vitro at low micromolar concentrations [60]. Furthermore, anti-edematous action has been described in a small series of brain tumor patients undergoing radio- or chemotherapy treated with the boswellia preparation H15 [61,62]. More recently, within a prospective trial 44 patients with primary or secondary malignant cerebral tumors were randomly assigned to receive radiotherapy plus either H15 or placebo. A reduction of cerebral edema of $>75 \%$ assessed by T2-weighted MRI was observed in $60 \%$ of patients treated with $\mathrm{H} 15$ compared to $26 \%$ of patients receiving placebo ( $\mathrm{p}=0.023)$. H15 was well tolerated except for minor gastrointestinal side effects in some patients. There was no significant difference in dexamethasone dose between the 2 groups. Furthermore, treatment with H15 had no significant impact on quality of life or cognitive function [63]. The exact mode of action of boswellic acids on peritumoral edema has remained unclear but may partially rely on an interference with the VEGF pathway and suppression of PGE2 formation [64,65]. Overall, larger studies are required to assess the potential steroid-sparing effects of boswellic acids and their impact on therapy-related edema in more detail. Furthermore, a better understanding of the molecular mechanisms underlying the functional activity of boswellia extracts may help to exploit their full therapeutic potential.

\section{COX-2 inhibitors}


COX-2 may contribute to the evolution of tumor-related edema as outlined above. Drugs which target COX-2 are widely available and have been partially assessed for their antiedematous efficacy. Celecoxib reduced VEGF expression levels in a preclinical tumor model [66]. The specific COX-2 inhibitor SC-236 was assessed in a rat brain tumor model and had a similar effect on survival than dexamethasone [67]. Rofecoxib inhibited the leakage of contrast medium in glioma-bearing rats to a similar amount as dexamethasone [20]. Whether these effects also occur in human patients and translate into clinical benefit has not been examined in detail. Case reports suggest a certain anti-edematous activity of COX-2 inhibition but confirmation in larger series is pending [68].

\section{Other agents}

Osmotherapeutic agents such as mannitol, glycerol, or hypertonic saline are frequently used in patients suffering from extensive brain edema after stroke or trauma. The activity of these agents against brain tumor-related edema has not been examined systematically. These drugs lead to osmotic diuresis which may cause severe shifts in electrolyte levels. Furthermore, the "rebound phenomenon", that is, edema recurrence upon treatment cessation, is a major limiting factor of their use. In a preclinical study, hypertonic saline given as a continuous intravenous infusion reduced the peritumoral edema in a rat brain tumor model [69]. However, because of the disrupted blood-brain barrier in many brain tumors, it must be considered that osmotherapeutics such as mannitol may leak into the brain parenchyma resulting in a further aggravation of the osmotic gradient [70]. Similarly, there are no data supporting a role for diuretics, e.g. furosemide, in the treatment of peritumoral edema. The use of osmotic agents should therefore be restricted to severely ill patients requiring immediate anti-edematous effects for a short period of time, e.g. during or after surgery. When steroids need to be avoided and this approach is considered clinically appropriate, e.g. in in patients with suspicion of lymphoma, osmotic agents may help to decrease edemaderived mass effect preoperatively until a tissue specimen has been obtained for histopathological assessment.

A small retrospective study analyzed the putative anti-edematous effects of angiotensin-II inhibitors in patients with newly diagnosed glioblastoma. It was reported that patients receiving angiotensin-II inhibitors, but not other antihypertensive drugs, required significantly 
lower doses of steroids during radiotherapy [71]. This observation may be worth being pursued within a prospective trial. Other experimental treatments directed against peritumoral edema have only been assessed in preclinical studies: argatroban, a thrombin antagonist, reduced the peritumoral edema in a rodent glioma model [72]. Glyburide, a well-known antidiabetic drug, inhibits the sulfonylurea receptor 1 and reduced cerebral metastasis-related edema in rats [73]. Furthermore, caloric restriction attenuated the tumor-surrounding edema in glioma-bearing mice [74]. Whether any of these approaches will ever be assessed within clinical trials, needs to be awaited.

\section{Expert commentary}

Reducing the clinical burden derived from edema-mediated mass effect is an important task in patients with primary and secondary brain tumors. Glucocorticoids are the most frequently used agents against tumor-surrounding edema. They provide rapid albeit transient relief from clinical symptoms due to edema-mediated elevated intracranial pressure. Owing to their significant side effects, tapering should be considered whenever regarded clinically feasible. Even after several decades of research, convincing strategies that may allow for a significant reduction of steroids are lacking. An improved understanding of the underlying molecular mechanisms involved in the formation of peritumoral edema may help to develop novel therapeutic approaches. Inhibition of VEGF which may be the major player in the development of edemas in brain cancers results frequently in a considerable anti-edema effect. However, anti-VEGF therapies are expensive and far away from representing convincing alternatives to steroids. Boswellic acids, in contrast, have a more favourable price level and bear mostly only minor side effects. Based on the results of smaller patient series and a more recent larger trial, it may be concluded that they have a certain steroid-sparing effect. Therefore, it might be warranted to assess boswellic acids and their anti-edema efficacy in larger studies.

\section{Five-year view}

Alternatives which may help to reduce the long-term administration of steroids are urgently needed in order to avoid their manifold side effects. Based on data published by Recht et al. 
[50], corticorelin acetate may currently be the most promising agent in this regard. However, several issues need further clarification such as the potential beneficial effects of the compound in patients who require rather small steroid doses. Whether corticorelin acetate in these patients may allow for a complete abstinence from steroids has not yet been examined. Furthermore, long-term toxicity as well as some safety issues, e.g. the combination with other compounds, is largely unknown. It remains to be seen whether the drug will be approved within the next years based on the available data. Further advances may arise from the ongoing research on $\mathrm{H} 15$ and similar boswellia serrata extracts. As with all other agents which have only been assessed within small series for their potential anti-edema activity, neurooncologists must strive for prospective, randomized-controlled trials in order to delineate their clinical use. Currently, no specific anti-edematous treatment based on the histology of the underlying tumor is available. A better understanding of the molecular mechanisms causing edema in different histological tumor entities may help to develop novel therapeutic strategies targeting edema.

\section{Key issues}

- Peritumoral edema can occur in virtually all CNS neoplasms and is most common in high-grade gliomas and metastases

- Several factors contribute to the development of vasogenic edema including the secretion of VEGF by tumor cells

- The mass effect derived from tumor-associated edema increases intracranial pressure and results in neurological symptoms, significantly contributing to morbidity

- Steroids are the mainstay of anti-edematous therapy. Their administration frequently leads to clinical improvement and symptom relief

- The mid- and long-term use of steroids is limited due to their numerous side effects such as metabolic changes, osteoporosis, myopathy, increased risk for opportunistic infections, psychiatric alterations and many more

- Corticorelin acetate, a synthetic analog of corticotropin releasing factor, may help to reduce steroids doses and steroid-related side effects

- Anti-angiogenic agents such as the VEGF inhibitor bevacizumab have pronounced anti-edema effects in many brain tumor patients 
- Several agents with putative anti-edematous activity have only been assessed in preclinical models or small patients series including COX-2 and angiotensin-II inhibitors and further clinical testing is required 


\section{References}

1. Osawa T, Tosaka M, Nagaishi M, Yoshimoto Y. Factors affecting peritumoral brain edema in meningioma: special histological subtypes with prominently extensive edema. J Neurooncol, 111(1), 49-57 (2013).

2. Wong JM, Panchmatia JR, Ziewacz JE et al. Patterns in neurosurgical adverse events: intracranial neoplasm surgery. Neurosurg Focus, 33(5), E16 (2012).

3. Bebawy JF. Perioperative steroids for peritumoral intracranial edema: a review of mechanisms, efficacy, and side effects. J Neurosurg Anesthesiol, 24(3), 173-177 (2012).

4. Unger KR, Lominska CE, Chanyasulkit J et al. Risk factors for posttreatment edema in patients treated with stereotactic radiosurgery for meningiomas. Neurosurgery, 70(3), 639-645 (2012).

5. Hirano A, Kawanami T, Llena JF. Electron microscopy of the blood-brain barrier in disease. Microsc Res Tech, 27(6), 543-556 (1994).

6. Pietsch T, Valter MM, Wolf HK et al. Expression and distribution of vascular endothelial growth factor protein in human brain tumors. Acta Neuropathol, 93(2), 109-117 (1997).

7. Senger DR, Galli SJ, Dvorak AM, Perruzzi CA, Harvey VS, Dvorak HF. Tumor cells secrete a vascular permeability factor that promotes accumulation of ascites fluid. Science, 219(4587), 983-985 (1983).

8. Strugar JG, Criscuolo GR, Rothbart D, Harrington WN. Vascular endothelial growth/permeability factor expression in human glioma specimens: correlation with vasogenic brain edema and tumor-associated cysts. J Neurosurg, 83(4), 682-689 (1995).

9. Provias J, Claffey K, delAguila L, Lau N, Feldkamp M, Guha A. Meningiomas: role of vascular endothelial growth factor/vascular permeability factor in angiogenesis and peritumoral edema. Neurosurgery, 40(5), 1016-1026 (1997).

10. Dobrogowska DH, Lossinsky AS, Tarnawski M, Vorbrodt AW. Increased blood-brain barrier permeability and endothelial abnormalities induced by vascular endothelial growth factor. J Neurocytol, 27(3), 163-173 (1998).

11. Ginat DT, Mangla R, Yeaney G, Schaefer PW, Wang H. Correlation between dynamic contrast-enhanced perfusion MRI relative cerebral blood volume and vascular 
endothelial growth factor expression in meningiomas. Acad Radiol, 19(8), 986-990 (2012).

12. Jain RK, di Tomaso E, Duda DG, Loeffler JS, Sorensen AG, Batchelor TT. Angiogenesis in brain tumours. Nat Rev Neurosci, 8(8), 610-622 (2007).

13. Chan AS, Leung SY, Wong MP et al. Expression of vascular endothelial growth factor and its receptors in the anaplastic progression of astrocytoma, oligodendroglioma, and ependymoma. Am J Surg Pathol, 22(7), 816-826 (1998).

14. Goldman CK, Bharara S, Palmer CA et al. Brain edema in meningiomas is associated with increased vascular endothelial growth factor expression. Neurosurgery, 40(6), 1269-1277 (1997).

15. Otsuka S, Tamiya T, Ono Y et al. The relationship between peritumoral brain edema and the expression of vascular endothelial growth factor and its receptors in intracranial meningiomas. J Neurooncol, 70(3), 349-357 (2004).

16. Nassehi D, Sorensen LP, Dyrbye H et al. Peritumoral brain edema in angiomatous supratentorial meningiomas: an investigation of the vascular endothelial growth factor A pathway. APMIS, Feb 11 [Epub ahead of print] (2013).

17. Black KL, Hoff JT, McGillicuddy JE, Gebarski SS. Increased leukotriene C4 and vasogenic edema surrounding brain tumors in humans. Ann Neurol, 19(6), 592-595 (1986).

18. Deininger MH, Weller M, Streffer J, Mittelbronn M, Meyermann R. Patterns of cyclooxygenase-1 and -2 expression in human gliomas in vivo. Acta Neuropathol, 98(3), 240-244 (1999).

19. Temel SG, Kahveci Z. Cyclooxygenase-2 expression in astrocytes and microglia in human oligodendroglioma and astrocytoma. J Mol Histol, 40(5-6), 369-377 (2009).

20. Badie B, Schartner JM, Hagar AR et al. Microglia cyclooxygenase-2 activity in experimental gliomas: possible role in cerebral edema formation. Clin Cancer Res, 9(2), 872-877 (2003).

21. Nathoo N, Barnett GH, Golubic M. The eicosanoid cascade: possible role in gliomas and meningiomas. J Clin Pathol, 57(1), 6-13 (2004).

22. Pistolesi S, Boldrini L, Gisfredi S et al. Expression of cyclooxygenase-2 and its correlation with vasogenic brain edema in human intracranial meningiomas. Cancer Invest, 25(7), 555-562 (2007). 
23. Mayhan WG. VEGF increases permeability of the blood-brain barrier via a nitric oxide synthase/cGMP-dependent pathway. Am J Physiol, 276(5 Pt 1), C1148-1153 (1999).

24. Broholm H, Rubin I, Kruse A et al. Nitric oxide synthase expression and enzymatic activity in human brain tumors. Clin Neuropathol, 22(6), 273-281 (2003).

25. Bulnes S, Argandona EG, Bengoetxea H, Leis O, Ortuzar N, Lafuente JV. The role of eNOS in vascular permeability in ENU-induced gliomas. Acta Neurochir Suppl, 106, 277-282 (2010).

26. Shibata S. Ultrastructure of capillary walls in human brain tumors. Acta Neuropathol, 78(6), 561-571 (1989).

27. Stewart PA, Hayakawa K, Hayakawa E, Farrell CL, Del Maestro RF. A quantitative study of blood-brain barrier permeability ultrastructure in a new rat glioma model. Acta Neuropathol, 67(1-2), 96-102 (1985).

28. Liebner S, Fischmann A, Rascher G et al. Claudin-1 and claudin-5 expression and tight junction morphology are altered in blood vessels of human glioblastoma multiforme. Acta Neuropathol, 100(3), 323-331 (2000).

29. Papadopoulos MC, Saadoun S, Woodrow CJ et al. Occludin expression in microvessels of neoplastic and non-neoplastic human brain. Neuropathol Appl Neurobiol, 27(5), 384-395 (2001).

30. Antonetti DA, Barber AJ, Hollinger LA, Wolpert EB, Gardner TW. Vascular endothelial growth factor induces rapid phosphorylation of tight junction proteins occludin and zonula occluden 1. A potential mechanism for vascular permeability in diabetic retinopathy and tumors. J Biol Chem, 274(33), 23463-23467 (1999).

31. Savaskan NE, Heckel A, Hahnen E et al. Small interfering RNA-mediated xCT silencing in gliomas inhibits neurodegeneration and alleviates brain edema. Nat Med, 14(6), 629-632 (2008).

32. Badaut J, Lasbennes F, Magistretti PJ, Regli L. Aquaporins in brain: distribution, physiology, and pathophysiology. J Cereb Blood Flow Metab, 22(4), 367-378 (2002).

33. Saadoun S, Papadopoulos MC, Davies DC, Bell BA, Krishna S. Increased aquaporin 1 water channel expression in human brain tumours. Br J Cancer, 87(6), 621-623 (2002).

34. Saadoun S, Papadopoulos MC, Davies DC, Krishna S, Bell BA. Aquaporin-4 expression is increased in oedematous human brain tumours. J Neurol Neurosurg Psychiatry, 72(2), 262-265 (2002). 
35. Warth A, Simon P, Capper D et al. Expression pattern of the water channel aquaporin4 in human gliomas is associated with blood-brain barrier disturbance but not with patient survival. J Neurosci Res, 85(6), 1336-1346 (2007).

36. Mou K, Chen M, Mao Q et al. AQP-4 in peritumoral edematous tissue is correlated with the degree of glioma and with expression of VEGF and HIF-alpha. J Neurooncol, 100(3), 375-383 (2010).

37. Lambertz N, Hindy NE, Adler C et al. Expression of aquaporin 5 and the AQP5 polymorphism $\mathrm{A}(-1364) \mathrm{C}$ in association with peritumoral brain edema in meningioma patients. J Neurooncol, 112(2), 297-305 (2013).

38. Ingraham FD, Matson DD, Mc LR. Cortisone and ACTH as an adjunct to the surgery of craniopharyngiomas. $N$ Engl J Med, 246(15), 568-571 (1952).

39. Galicich JH, French LA, Melby JC. Use of dexamethasone in treatment of cerebral edema associated with brain tumors. J Lancet, 81, 46-53 (1961).

40. Roth P, Wick W, Weller M. Steroids in neurooncology: actions, indications, sideeffects. Curr Opin Neurol, 23(6), 597-602 (2010).

41. Ryan R, Booth S, Price S. Corticosteroid-use in primary and secondary brain tumour patients: a review. J Neurooncol, 106(3), 449-459 (2012).

42. Vale W, Spiess J, Rivier C, Rivier J. Characterization of a 41-residue ovine hypothalamic peptide that stimulates secretion of corticotropin and beta-endorphin. Science, 213(4514), 1394-1397 (1981).

43. Reubi JC, Waser B, Vale W, Rivier J. Expression of CRF1 and CRF2 receptors in human cancers. J Clin Endocrinol Metab, 88(7), 3312-3320 (2003).

44. Tjuvajev J, Uehara H, Desai R et al. Corticotropin-releasing factor decreases vasogenic brain edema. Cancer Res, 56(6), 1352-1360 (1996).

45. Wei ET, Gao GC. Corticotropin-releasing factor: an inhibitor of vascular leakage in rat skeletal muscle and brain cortex after injury. Regul Pept, 33(2), 93-104 (1991).

46. Moliterno JA, Henry E, Pannullo SC. Corticorelin acetate injections for the treatment of peritumoral brain edema. Expert Opin Investig Drugs, 18(9), 1413-1419 (2009).

47. Villalona-Calero MA, Eckardt J, Burris $\mathrm{H}$ et al. A phase I trial of human corticotropinreleasing factor (hCRF) in patients with peritumoral brain edema. Ann Oncol, 9(1), 7177 (1998).

48. Moroz MA, Huang R, Kochetkov T et al. Comparison of corticotropin-releasing factor, dexamethasone, and temozolomide: treatment efficacy and toxicity in U87 and C6 intracranial gliomas. Clin Cancer Res, 17(10), 3282-3292 (2011). 
49. Gamez I, Ryan RP, Keir ST. Corticorelin acetate, a synthetic corticotropin-releasing factor with preclinical antitumor activity, alone and with bevacizumab, against human brain tumor models. Anticancer Res, 30(12), 5037-5042 (2010).

50. Recht L, Mechtler LL, Wong ET, O'Connor PC, Rodda BE. Steroid-sparing effect of corticorelin acetate in peritumoral cerebral edema is associated with improvement in steroid-induced myopathy. J Clin Oncol, 31(9), 1182-1187 (2013).

** A prospective, randomized, double-blind trial suggesting that corticorelin acetate allows for a reduction of steroid administration and results in a reduction in the incidence and severity of some steroid side effects

51. Berkman RA, Merrill MJ, Reinhold WC et al. Expression of the vascular permeability factor/vascular endothelial growth factor gene in central nervous system neoplasms. $J$ Clin Invest, 91(1), 153-159 (1993).

52. Vredenburgh JJ, Cloughesy T, Samant M et al. Corticosteroid use in patients with glioblastoma at first or second relapse treated with bevacizumab in the BRAIN study. Oncologist, 15(12), 1329-1334 (2010).

* An analysis of the BRAIN trial demonstrating that bevacizumab may have corticosteroidsparing effects in patients with recurrent glioblastoma

53. Gerstner ER, Duda DG, di Tomaso E et al. VEGF inhibitors in the treatment of cerebral edema in patients with brain cancer. Nat Rev Clin Oncol, 6(4), 229-236 (2009).

54. Ellingson BM, Cloughesy TF, Lai A et al. Quantification of edema reduction using differential quantitative T2 (DQT2) relaxometry mapping in recurrent glioblastoma treated with bevacizumab. J Neurooncol, 106(1), 111-119 (2012).

55. Kamoun WS, Ley CD, Farrar CT et al. Edema control by cediranib, a vascular endothelial growth factor receptor-targeted kinase inhibitor, prolongs survival despite persistent brain tumor growth in mice. J Clin Oncol, 27(15), 2542-2552 (2009).

* Preclinical evidence on the anti-edema effect of cediranib in a rodent glioma model

56. Batchelor TT, Sorensen AG, di Tomaso E et al. AZD2171, a pan-VEGF receptor tyrosine kinase inhibitor, normalizes tumor vasculature and alleviates edema in glioblastoma patients. Cancer Cell, 11(1), 83-95 (2007).

57. Batchelor TT, Duda DG, di Tomaso E et al. Phase II study of cediranib, an oral panvascular endothelial growth factor receptor tyrosine kinase inhibitor, in patients with recurrent glioblastoma. J Clin Oncol, 28(17), 2817-2823 (2010). 
58. Batchelor T, Mulholland P, Neyns B et al. The efficacy of cediranib as monotherapy and in combination with lomustine compared to lomustine alone in patients with recurrent glioblastoma: a phase III randomized study. Neuro Oncol 12 (suppl 4), iv69iv78 (2010).

59. Iwamoto FM, Lamborn KR, Robins HI et al. Phase II trial of pazopanib (GW786034), an oral multi-targeted angiogenesis inhibitor, for adults with recurrent glioblastoma (North American Brain Tumor Consortium Study 06-02). Neuro Oncol, 12(8), 855$861(2010)$.

60. Glaser T, Winter S, Groscurth $\mathrm{P}$ et al. Boswellic acids and malignant glioma: induction of apoptosis but no modulation of drug sensitivity. Br J Cancer, 80(5-6), 756-765 (1999).

61. Janssen G, Bode U, Breu H, Dohrn B, Engelbrecht V, Gobel U. Boswellic acids in the palliative therapy of children with progressive or relapsed brain tumors. Klin Padiatr, 212(4), 189-195 (2000).

62. Streffer JR, Bitzer M, Schabet M, Dichgans J, Weller M. Response of radiochemotherapy-associated cerebral edema to a phytotherapeutic agent, H15. Neurology, 56(9), 1219-1221 (2001).

63. Kirste S, Treier M, Wehrle SJ et al. Boswellia serrata acts on cerebral edema in patients irradiated for brain tumors: a prospective, randomized, placebo-controlled, double-blind pilot trial. Cancer, 117(16), 3788-3795 (2011).

** A randomized, placebo-controlled, double-blind trial demonstrating the anti-edema activity of boswellic acids

64. Pang X, Yi Z, Zhang X et al. Acetyl-11-keto-beta-boswellic acid inhibits prostate tumor growth by suppressing vascular endothelial growth factor receptor 2-mediated angiogenesis. Cancer Res, 69(14), 5893-5900 (2009).

* This study suggests a molecular mechanism which may underlie the anti-edema activity of boswellic acids

65. Siemoneit U, Koeberle A, Rossi A et al. Inhibition of microsomal prostaglandin E2 synthase- 1 as a molecular basis for the anti-inflammatory actions of boswellic acids from frankincense. Br J Pharmacol, 162(1), 147-162 (2011).

66. Wei D, Wang L, He Y, Xiong HQ, Abbruzzese JL, Xie K. Celecoxib inhibits vascular endothelial growth factor expression in and reduces angiogenesis and metastasis of human pancreatic cancer via suppression of Sp1 transcription factor activity. Cancer Res, 64(6), 2030-2038 (2004). 
67. Portnow J, Suleman S, Grossman SA, Eller S, Carson K. A cyclooxygenase-2 (COX2) inhibitor compared with dexamethasone in a survival study of rats with intracerebral 9L gliosarcomas. Neuro Oncol, 4(1), 22-25 (2002).

68. Khan RB, Krasin MJ, Kasow K, Leung W. Cyclooxygenase-2 inhibition to treat radiation-induced brain necrosis and edema. J Pediatr Hematol Oncol, 26(4), 253-255 (2004).

69. Toung TJ, Tyler B, Brem H, Traystman RJ, Hurn PD, Bhardwaj A. Hypertonic saline ameliorates cerebral edema associated with experimental brain tumor. J Neurosurg Anesthesiol, 14(3), 187-193 (2002).

70. Palma L, Bruni G, Fiaschi AI, Mariottini A. Passage of mannitol into the brain around gliomas: a potential cause of rebound phenomenon. A study on 21 patients. $J$ Neurosurg Sci, 50(3), 63-66 (2006).

71. Carpentier AF, Ferrari D, Bailon O et al. Steroid-sparing effects of angiotensin-II inhibitors in glioblastoma patients. Eur J Neurol, 19(10), 1337-1342 (2012).

72. Hua Y, Keep RF, Schallert T, Hoff JT, Xi G. A thrombin inhibitor reduces brain edema, glioma mass and neurological deficits in a rat glioma model. Acta Neurochir Suppl, 86, 503-506 (2003).

73. Thompson EM, Pishko GL, Muldoon LL, Neuwelt EA. Inhibition of SUR1 decreases the vascular permeability of cerebral metastases. Neoplasia, 15(5), 535-543 (2013).

74. Jiang YS, Wang FR. Caloric restriction reduces edema and prolongs survival in a mouse glioma model. J Neurooncol, May 24. [Epub ahead of print] (2013). 